Acta Crystallographica Section D

\section{Biological Crystallography}

ISSN 0907-4449

\section{Günter Pappenberger,‡ Tanja Schulz-Gasch, Eric Kusznir, Francis Müller and Michael Hennig*}

F. Hoffmann-La Roche Ltd, Pharma Research Discovery, CH-4070 Basel, Switzerland

‡ Current address: DSM Nutritional Products Ltd, R\&D Center for Biotechnology, PO Box 3255, CH-4002 Basel, Switzerland.

Correspondence e-mail:

michael.hennig@roche.com

\title{
Structure-assisted discovery of an aminothiazole derivative as a lead molecule for inhibition of bacterial fatty-acid synthesis
}

Fatty-acid synthesis in bacteria is of great interest as a target for the discovery of antibacterial compounds. The addition of a new acetyl moiety to the growing fatty-acid chain, an essential step in this process, is catalyzed by $\beta$-ketoacyl-ACP synthase (KAS). It is inhibited by natural antibiotics such as cerulenin and thiolactomycin; however, these lack the requirements for optimal drug development. Structure-based biophysical screening revealed a novel synthetic small molecule, 2-phenylamino-4-methyl-5-acetylthiazole, that binds to Escherichia coli KAS I with a binding constant of $25 \mu M$ as determined by fluorescence titration. A $1.35 \AA$ crystal structure of its complex with its target reveals noncovalent interactions with the active-site Cys163 and hydrophobic residues of the fatty-acid binding pocket. The active site is accessible through an open conformation of the Phe392 side chain and no conformational changes are induced at the active site upon ligand binding. This represents a novel binding mode that differs from thiolactomycin or cerulenin interaction. The structural information on the protein-ligand interaction offers strategies for further optimization of this low-molecularweight compound.

\section{Introduction}

Antibacterial therapeutics are of great medical importance and have saved countless human lives in past decades. Owing to the rise in drug resistance amongst major human pathogens in recent years, there is an urgent need for antibiotics with novel mechanisms of action (Barrett \& Barrett, 2003). One promising target for the discovery of such new antibacterial therapeutics is the biosynthesis of fatty acids in bacteria (Heath et al., 2001; Payne, 2004). Fatty-acid biosynthesis is an essential and highly conserved enzymatic process. The growing fatty-acid chain is shuttled between the catalytic functions while bound to a small acyl carrier protein (ACP) via a phosphopantetheine linker. The high degree of conservation in fatty-acid biosynthesis creates the potential for a genuinely broad-spectrum antibiotic. On the other hand, significant differences between prokaryotic and eukaryotic (and in particular mammalian) fatty-acid biosynthesis will allow the necessary differentiation towards the prokaryotic target. In prokaryotes the series of catalytic steps for fatty-acid biosynthesis is performed by individual enzymes (type II fattyacid biosynthesis; Rock \& Jackowski, 2002), while in mammals all the required catalytic functions are united on a large single polypeptide chain (type I fatty-acid biosynthesis; Smith et al., 2003). The success of fatty-acid biosynthesis as an antibacterial target is exemplified by triclosan, a chlorinated bisphenol that selectively inhibits bacterial enoyl-ACP reductase. It has been in use for decades as an additive in many consumer products
Received 9 August 2007

Accepted 10 October 2007

PDB References: $\beta$-ketoacylACP synthase, 2vb7, r2vb7sf; 2vb8, r2vb8sf; 2vb9, r2vb9sf; $2 \mathrm{vba}, \mathrm{r} 2 \mathrm{vbasf}$. 
owing to its bactericidal properties (Bhargava \& Leonard, 1996).

Another highly interesting target within the fatty-acid biosynthetic pathway is $\beta$-ketoacyl-ACP synthase (KAS; Khandekar et al., 2003). This enzyme catalyses the Claisen condensation reaction, i.e. carbon-carbon bond formation upon the addition of a new acetyl unit to the growing fattyacid chain (Heath \& Rock, 2002). The reaction can be divided into three distinct steps: (i) transfer of the acyl group from acyl-ACP to the active-site cysteine residue, resulting in a thioester, (ii) binding of malonyl-ACP and subsequent decarboxylation to form a carbanion and (iii) nucleophilic attack of the carbanion on the carbonyl carbon of the thioester to form the carbon-carbon bond. The functional oligomer of the KAS protein is the homodimer, with residues from both monomers being involved in each fatty-acid-binding pocket (Edwards et al., 1997; Huang et al., 1998; Olsen et al., 1999). Three related but distinct enzymes with KAS activity are found in Escherichia coli and some other bacteria (KAS I, II and III, also known as FabB, FabF and FabH, respectively; Heath \& Rock, 2002). KAS I and KAS III are essential enzymes in E. coli, in contrast to KAS II (Rosenfeld et al., 1973; Garwin et al., 1980; Lai \& Cronan, 2003).

Several novel inhibitors of bacterial KAS III protein have recently been reported (He \& Reynolds, 2002; Daines et al., 2003; He et al., 2004; Nie et al., 2005), highlighting the ongoing efforts to target novel antibacterial drugs against KAS activity. KAS I is a particularly attractive target as it has already been validated through the action of two compounds from natural sources: cerulenin and thiolactomycin (Fig. 1). Both inhibit KAS I and KAS II but not KAS III activity (Price et al., 2001). Cerulenin (from Cephalosporium caerulens) has a reactive epoxy group that forms a covalent adduct with the active-site cysteine of KAS (Price et al., 2001; Vance et al., 1972; D'Agnolo et al., 1973; Moche et al., 1999). It is not a selective antibacterial, however, since it also inhibits the KAS function of the mammalian FAS multienzyme complex. The second compound, thiolactomycin, is a thiolactone from actino-
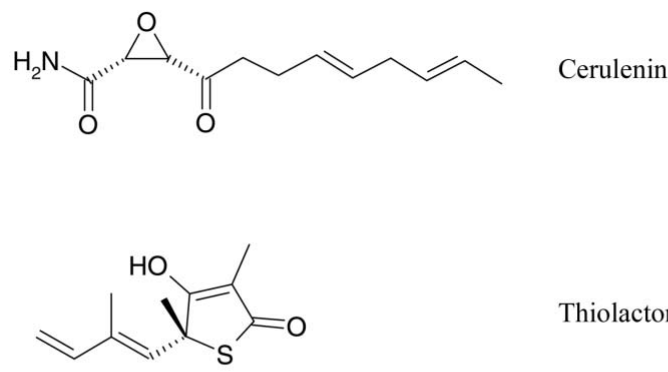

Thiolactomycin

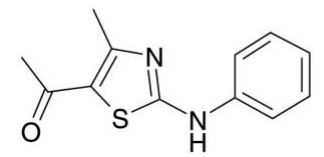

2-Phenylamino-4-methyl5-acetylthiazole

Figure 1

Structures of cerulenin, thiolactomycin and 2-phenylamino-4-methyl-5acetylthiazole. bacteria (Nocardia sp.) that reversibly inhibits bacterial KAS (Price et al., 2001; Nishida et al., 1986). It is active against a wide range of bacteria and other pathogens such as plasmodia and trypanosomes (Noto et al., 1982; Waller et al., 1998; Morita et al., 2000). It does not affect mammalian fatty-acid biosynthesis (Hayashi et al., 1983) and in mice it protects against infections without toxic side effects (Miyakawa et al., 1982). However, problems in synthesis and with stability have prevented its use as an antibiotic drug (Heath et al., 2001). Thiolactomycin-derived compounds with improved properties are currently being explored as inhibitors of bacterial and mammalian KAS (Sakya et al., 2001; Douglas et al., 2002; McFadden et al., 2005). Recently, a novel compound from a natural source, platensimycin, was discovered to be a potent inhibitor of bacterial KAS I and KAS II, with antibiotic properties in animal models (Wang et al., 2006). However, it remains challenging to develop such complex natural products into drug molecules with the required efficacy, synthetic access and pharmacological properties.

Here, we describe for the first time a small synthetic compound that binds to the KAS I active site. The interaction of this aminothiazole derivative with E. coli KAS I has an equilibrium dissociation constant of $25 \mu \mathrm{M}$. A high-resolution crystal structure of the protein-ligand complex at $1.35 \AA$ could be obtained after optimization of the buffer conditions in order to improve the solubility of the ligand. The structure provides insights into the binding mode and suggests strategies for further optimization of this inhibitor.

\section{Materials and methods}

\subsection{Binding assay and ligand solubility by analytical ultracentrifugation}

Sedimentation-equilibrium runs were performed at a rotational speed of $16000 \mathrm{rev} \mathrm{min}^{-1}$ at $293 \mathrm{~K}$ using a BeckmanCoulter Optima XL-I analytical ultracentrifuge. An eight-hole rotor fitted with six-channel Alu-filled Epon analytical cells was used. The reference channels were filled with $120 \mu \mathrm{l}$ binding-assay buffer ( $20 \mathrm{~m} M$ HEPES pH 7.5, $200 \mathrm{~m} M \mathrm{NaCl}$, $2 \mathrm{~m} M$ TCEP, $0.5 \mathrm{~m} M$ EDTA), whereas the sample channels were filled with $100 \mu$ buffer solution containing $22.7 \mu M$ $\left(1 \mathrm{mg} \mathrm{ml}^{-1}\right)$ E. coli KAS I and $100 \mu M$ ligand. Cells with protein and ligand alone at the above concentrations were also spun as a control. After $20 \mathrm{~h}$, a radial absorbance profile was recorded at $280 \mathrm{~nm}$. A further recording taken $2 \mathrm{~h}$ later proved that equilibrium had been reached. Equilibrium absorbance profiles were then recorded at the specific wavelengths of the studied ligands.

Analysis of the profiles was performed using the program DISCREEQ (Schuck, 1994). Analysis of the optical density at the ligand characteristic wavelength yields, with the help of the absorption coefficient, the amount of bound ligand, the stoichiometry, the free ligand concentration through baseline analysis and ligand-induced protein precipitation if it occurs.

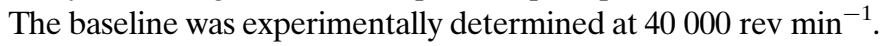
The ligand absorption coefficients were determined from 
absorption spectra recorded on an Uvikon 930 spectrophotometer. The absorption coefficient of the protein at $280 \mathrm{~nm}$ was calculated from the amino-acid sequence. For other wavelengths it was derived from a wavelength scan recorded at $3000 \mathrm{rev} \mathrm{min}^{-1}$ at the beginning of the equilibrium experiment.

The radial absorbance profiles of the low-molecular-weight aminothiazole compound were recorded at $380 \mathrm{~nm}$. At this wavelength only the compound was detected, the protein absorption being negligible. In this case, visual inspection of the recorded absorbance profile readily revealed binding because at the chosen speed the profile for the ligand alone is flat (Fig. 2b).

Ligand solubility in the buffer of interest was assessed by recording the sedimentation equilibrium in a further higher speed run $\left(40000 \mathrm{rev} \mathrm{min}^{-1}\right)$ for a channel containing the ligand alone. The solubility was calculated from the area under the absorbance profile at the ligand specific wavelength, which was $380 \mathrm{~nm}$ for the aminothiazole (data not shown).

\subsection{Fluorescence titration}

Fluorescence titration was performed at $293 \mathrm{~K}$ in bindingassay buffer using an SLM-AMINCO 8100 double-grating spectrofluorometer. The protein concentration was $4.9 \mu \mathrm{M}$. Small aliquots of known ligand concentration were added and the fluorescence, excited at $280 \mathrm{~nm}$, was recorded at $340 \mathrm{~nm}$. The fluorescence intensity was corrected for protein dilution and for the filter effect (Birdsall et al., 1983). The corrected fluorescence intensity was plotted against the ligand concentration and fitted using a four-parameter sigmoidal function, from which the equilibrium dissociation constant $K_{\mathrm{d}}$ was computed using the law of mass action assuming a 1:1 proteinligand complex (Eftink, 1997).

\subsection{Cloning, expression, purification and crystallization}

The cloning, expression and purification of E. coli KAS I was performed essentially as described previously (Edwards $e t$ al., 1997). Briefly, the gene for E. coli KAS I was amplified from genomic $E$. coli DNA and cloned into the vector pQE- 80 via Bam $\mathrm{HI}$ and SalI. The protein construct with an $\mathrm{N}$-terminal His tag was expressed in E. coli M15 strain. The expression yield was high, with about $50 \%$ of the $E$. coli KAS I present in the soluble supernatant. Efficient purification of $E$. coli KAS I was achieved using immobilized metal-affinity chromatography (IMAC; Ni-NTA matrix) and size-exclusion chromatography (SEC; S75 matrix). Purification progress was assessed using SDS-PAGE. The purified protein was concentrated to $70 \mathrm{mg} \mathrm{ml}^{-1}$ in $20 \mathrm{~m} M$ HEPES pH 7.5, $200 \mathrm{mM}$ $\mathrm{NaCl}, 2 \mathrm{~m} M$ TCEP, $0.5 \mathrm{~m} M$ EDTA, flash-frozen in liquid nitrogen in $200 \mu \mathrm{l}$ aliqouts and stored at 193 K. E. coli KAS I was crystallized at room temperature in a hanging-drop experiment with $1.9 \mathrm{M}$ ammonium sulfate, $3 \%$ PEG 400 and $0.1 M$ Tris- $\mathrm{HCl} \mathrm{pH} 7.5$ as precipitant (Olsen et al., 1995). The protein concentration was $70 \mathrm{mg} \mathrm{ml}^{-1}$ in $20 \mathrm{~m} M$ HEPES $\mathrm{pH}$ 7.5, $200 \mathrm{~m} M \mathrm{NaCl}, 2 \mathrm{~m} M$ TCEP, $0.5 \mathrm{~m} M$ EDTA. The mixing ratio of buffer to protein was 2:1. Crystals appeared after 1-2 d and grew to full size after a few days. Crystals were soaked with thiolactomycin for $16-24 \mathrm{~h}$ after transfer into $2.3 \mathrm{M}$ ammonium sulfate, $0.1 M$ Tris- $\mathrm{HCl} \mathrm{pH} 7.5,0.3 \mathrm{~m} M$ thiolactomycin, 3\% DMSO. For soaking with the aminothiazole under PEG 8000 conditions, a $20 \mu \mathrm{l}$ drop of $30 \%$ PEG 8000, $0.1 M$ ammonium sulfate, $0.1 M$ Tris- $\mathrm{HCl}$ pH 7.5, $5 \mathrm{~m} M$ TCEP was equilibrated overnight in a hanging-drop setup against $0.5 \mathrm{ml} 2.3 \mathrm{M}$ ammonium sulfate, $0.1 M$ Tris- $\mathrm{HCl} \mathrm{pH}$ 7.5. The aminothiazole compound was added to the drop to $5 \mathrm{~m} M$ from a $100 \mathrm{~m} M$ DMSO stock (5\% residual DMSO) and crystals

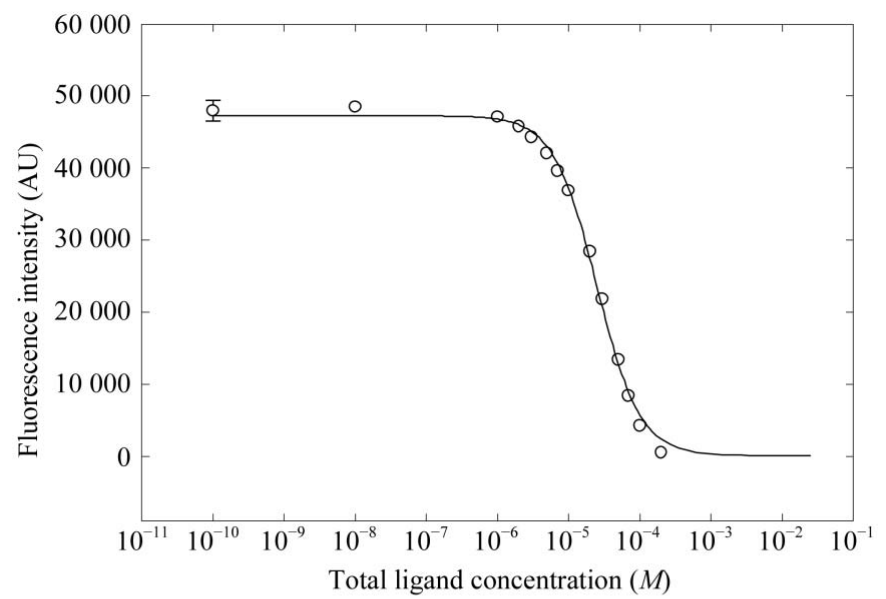

(a)

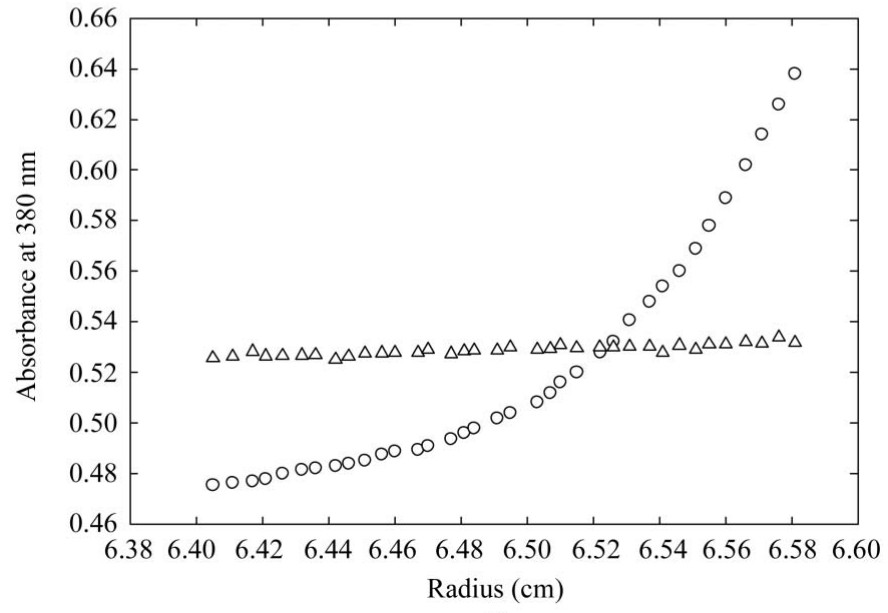

(b)

Figure 2

(a) Fluorescence titration of the aminothiazole ligand against E. coli KAS I protein. The protein concentration was $4.9 \mu M$ in binding-assay buffer (20 $\mathrm{m} M$ Tris pH 7.5, $200 \mathrm{~m} M \mathrm{NaCl}, 1 \mathrm{~m} M$ TCEP, $0.5 \mathrm{~m} M$ EDTA). The excitation and emission wavelengths were 280 and $340 \mathrm{~nm}$, respectively. Owing to the high absorption coefficient of the compound at $340 \mathrm{~nm}$, the efficient dipole-dipole interaction strongly quenched the emission of both tryptophan residues of the protein. Circles show the fluorescence intensity in arbitrary units after correction for filter effects; the line shows a sigmoidal fit resulting in an equilibrium dissociation constant $K_{\mathrm{d}}$ of $25 \mu M$. (b) Sedimentation equilibrium of the aminothiazole compound in the absence and presence of $E$. coli KAS I. The absorbance profiles were recorded at $16000 \mathrm{rev} \mathrm{min}^{-1}$ at $380 \mathrm{~nm}$ for $100 \mu \mathrm{M}$ aminothiazole in the absence or presence of $22.7 \mu M$ E. coli KAS I. At this wavelength the absorbance of the protein is negligible, so that only the ligand was detected. The ligand alone showed a flat radial profile for this rotational speed (triangles). In the presence of $E$. coli KAS I the radial concentration profile corresponds to the protein molar mass, demonstrating binding of the ligand (circles). 
Table 1

Data-collection and refinement statistics.

Values in parentheses are for the highest resolution shell.

\begin{tabular}{|c|c|c|c|c|}
\hline & $\begin{array}{l}\text { Apo in } \\
\left(\mathrm{NH}_{4}\right)_{2} \mathrm{SO}_{4}\end{array}$ & $\begin{array}{l}\text { Apo in } \\
\text { PEG } 8000\end{array}$ & $\begin{array}{l}\text { Thiolactomycin } \\
\text { in }\left(\mathrm{NH}_{4}\right)_{2} \mathrm{SO}_{4}\end{array}$ & $\begin{array}{l}\text { Aminothiazole } \\
\text { in PEG } 8000\end{array}$ \\
\hline PDB code & $2 \mathrm{vb} 9$ & $2 \mathrm{vb7}$ & $2 \mathrm{vb} 8$ & $2 \mathrm{vba}$ \\
\hline \multicolumn{5}{|l|}{ Data collection } \\
\hline Space group & $P 2_{1} 2_{1} 2_{1}$ & $P 2_{1} 2_{1} 2_{1}$ & $P 2_{1} 2_{1} 2_{1}$ & $P 2_{1} 2_{1} 2_{1}$ \\
\hline \multicolumn{5}{|c|}{ Unit-cell parameters } \\
\hline$a(\AA 0 \cap)$ & 58.9 & 59.1 & 59.1 & 59.0 \\
\hline$b(\AA)$ & 138.4 & 139.5 & 138.4 & 139.5 \\
\hline$c(\AA)$ & 211.9 & 212.2 & 212.0 & 212.2 \\
\hline$\alpha=\beta=\gamma\left({ }^{\circ}\right)$ & 90 & 90 & 90 & 90 \\
\hline Resolution (A) & $20-1.50(1.60-1.50)$ & $20-1.60(1.70-1.60)$ & $20-1.50(1.60-1.50)$ & $20-1.35(1.45-1.35)$ \\
\hline$R_{\text {merge }}$ & $7.5(48.4)$ & $7.4(22.3)$ & $6.1(37.2)$ & $5.8(20.4)$ \\
\hline$I / \sigma(I)$ & $12.0(3.0)$ & $10.9(5.3)$ & $14.2(3.5)$ & $13.5(6.2)$ \\
\hline Completeness (\%) & $99.4(98.0)$ & $95.0(75.4)$ & $95.8(78.0)$ & $92.3(72.9)$ \\
\hline Redundancy & $7.1(6.1)$ & $6.9(4.2)$ & $6.3(5.4)$ & $6.8(5.2)$ \\
\hline \multicolumn{5}{|l|}{ Refinement } \\
\hline Resolution $(\AA)$ & $20-1.50$ & $20-1.60$ & $20-1.50$ & $20-1.35$ \\
\hline No. of reflections & 262111 & 210551 & 253711 & 337314 \\
\hline$R_{\mathrm{work}} / R_{\text {free }}$ & $19.7 / 23.8$ & $15.6 / 18.6$ & $15.1 / 18.5$ & $11.9 / 15.1$ \\
\hline \multicolumn{5}{|l|}{ No. of atoms } \\
\hline Total & 14240 & 14475 & 14649 & 14386 \\
\hline Protein & 11903 & 11947 & 11946 & 11939 \\
\hline Ligand & - & - & 56 & 16 \\
\hline Water/ion & 2333 & 2528 & 2645 & 2430 \\
\hline \multicolumn{5}{|l|}{$B$ factors $\left(\AA^{2}\right)$} \\
\hline Overall & 20.2 & 17.9 & 16.8 & 17.5 \\
\hline Protein & 17.5 & 14.9 & 13.5 & 14.6 \\
\hline Ligand & - & - & 16.1 & 26.3 \\
\hline Water/ion & 36.2 & 33.7 & 34.0 & 33.3 \\
\hline \multicolumn{5}{|l|}{ R.m.s. deviations } \\
\hline Bond lengths $(\AA)$ & 0.015 & 0.012 & 0.015 & 0.014 \\
\hline Bond angles $\left({ }^{\circ}\right)$ & 1.53 & 1.36 & 1.50 & 1.58 \\
\hline
\end{tabular}

symmetry (NCS) restraints. Individual isotropic $B$ factors were refined in $R E F M A C$ for all data sets, with the exception of the structure with aminothiazole ligand bound. Here, individual anisotropic $B$ factors were introduced in the final round of refinement, resulting in a decrease in $R_{\text {free }}$ from 0.179 to 0.151 . The refinement statistics are reported in Table 1. Figures were prepared using $P y M O L$ (DeLano, 2002).

\subsection{Molecular modelling}

ROCS v.2.1.1 (Rapid Overlay of Chemical Structures, Openeye Scientific Software) was applied in order to investigate the shape and chemical similarity of the aminothiazole compound and thiolactomycin. The conformation of thiolactomycin was taken from the E. coli KAS I-thiolactomycin crystal structure. For the aminothiazole compound, OMEGA v.1.8.1 (Openeye Scientific Software) was used to generate

were soaked in this drop for 16-24 h. For the apo structure from PEG 8000 conditions, DMSO without the aminothiazole was added to the drop to $5 \%$ and the TCEP was omitted from the drop. Crystals were frozen in $2.3 \mathrm{M}$ ammonium sulfate, $0.1 M$ Tris- $\mathrm{HCl} \mathrm{pH} \mathrm{7.5,25 \%} \mathrm{glycerol} \mathrm{for} \mathrm{the} \mathrm{ammonium}$ sulfate conditions and directly from the drop for the PEG 8000 conditions.

\subsection{Data collection, processing and refinement}

Diffraction data for all data sets were collected at $120 \mathrm{~K}$ at the Swiss Light Source (SLS), Villigen, Switzerland. The diffraction images were processed using $X D S$ and scaled with XSCALE (Kabsch, 1993). No significant difference in unit-cell parameters was observed between crystals soaked in the ammonium sulfate and PEG 8000 conditions (Table 1). The structure was solved by molecular replacement using $E$. coli KAS I (PDB code 1ek4; Olsen et al., 2001) as a model. Rigidbody refinement of the four monomers followed by restrained refinement in REFMAC (Murshudov et al., 1999; Collaborative Computational Project, Number 4, 1994) and $A R P / w A R P$ v.5.0 (Perrakis et al., 1999) was used for refinement and to add water molecules to the model. Ligand building in the $F_{\mathrm{o}}-F_{\mathrm{c}}$ difference electron-density maps and manual rebuilding were performed with MOLOC (Gerber, 1992). The high resolution of the data sets (1.35-1.60 $⿱$ ) enabled the refinement of alternative side-chain conformations for several side chains. Final refinement was performed without noncrystallographic multiple low-energy conformations. Default settings were used in the ROCS and OMEGA calculations, except for turning on the ImplicitMillsDean flag for the colour score in $R O C S$. The final scores derived were a shape Tanimoto score for shape similarity (between 0 and 1 ) and a scaled colour score for chemical similarity (between 0 and 1). FRED v.1.2.10 (Fast Rigid Exhaustive Docking, Openeye Scientific Software) was used as a docking tool to investigate the binding mode of the aminothiazole compound to $E$. coli KAS I. Docking was performed using the structure of the apoprotein with water molecules removed. The binding site was defined as a box around the positions of thiolactomycin and cerulenin and increased by $2 \AA$. ChemScore was used as a scoring function.

\section{Results and discussion}

\subsection{Identification of novel molecules for the inhibition of E. coli KAS I}

For the E. coli KAS I protein, structural information is available for the apoprotein and for several protein-inhibitor complexes (Olsen et al., 1999, 2001; Price et al., 2001). Based on this knowledge, an in silico screening was performed with the goal of identifying novel hits for exploration in a drugdiscovery program. About 100 hits were selected based on compound availability, synthetic feasibility and drug-likeness to be tested experimentally for binding to E. coli KAS I by analytical ultracentrifugation. This method is independent of 
the biochemistry of the target, requires essentially no targetspecific assay development, has no requirements for labelling or immobilization methods and has a moderate throughput of 20-30 compounds per day. For one of the compounds, an aminothiazole derivative (2-phenylamino-4-methyl-5-acetyl-

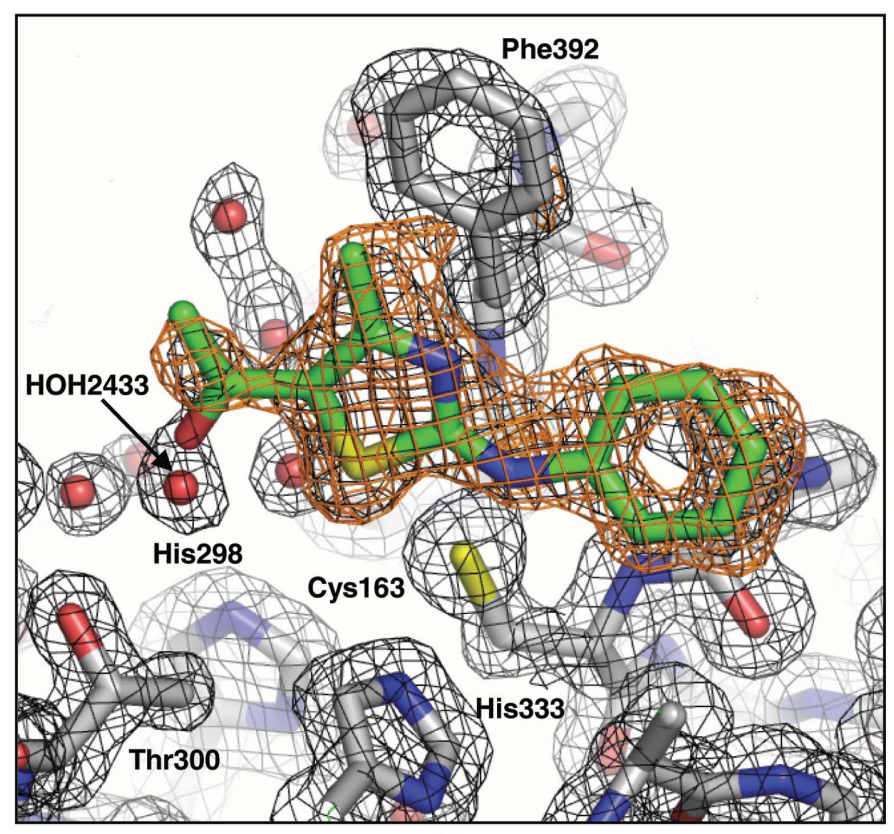

(a)

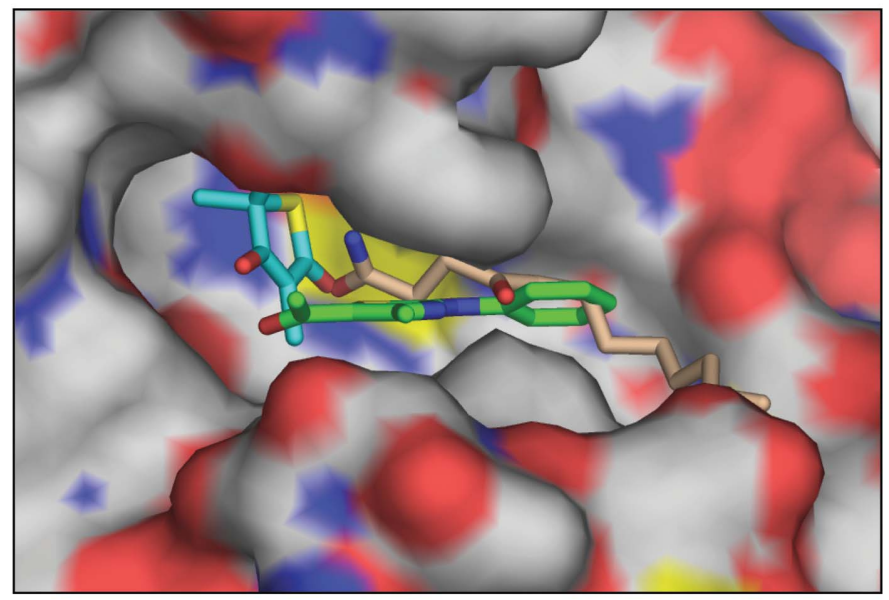

(b)

\section{Figure 3}

Binding mode of the aminothiazole ligand to E. coli KAS I protein. (a) Electron density of the aminothiazole ligand (green) bound to the active site of $E$. coli KAS I protein (grey). The $2 F_{\mathrm{o}}-F_{\mathrm{c}}$ OMIT map (contoured at $1 \sigma$ ) is shown in black; the $F_{\mathrm{o}}-F_{\mathrm{c}}$ OMIT map (contoured at $2.5 \sigma$ ) is shown in orange. The OMIT map in the absence of inhibitor was calculated using the final refined model. (b) Superposition of the binding modes for the KAS I ligands thiolactomycin (cyan), cerulenin (gold) and aminothiazole (green). For the representation of the protein, the structure with bound aminothiazole ligand was used. The protein is shown in surface representation, coloured according to atom type (C, grey; N, blue; $\mathrm{O}$, red; $\mathrm{S}$, yellow). Monomer $B$ of the dimer has been removed, as well as side chains Met204 and Val270 of monomer $A$, to enable insight into the binding pocket. With an intact binding site, only the acetyl moiety of the aminothiazole ligand is exposed to solvent. The isoprenoid tail of thiolactomycin is buried in a hydrophobic pocket and is not visible in this representation. The structure and binding mode of cerulenin was taken from PDB entry 1fj8 (Price et al., 2001). thiazole; Fig. 1), a clear sign of co-sedimentation with the protein was detected. The binding affinity of this novel ligand to $E$. coli KAS I was further evaluated by fluorescence titration. Upon addition of the aminothiazole ligand to the protein in solution, the inherent tryptophan fluorescence of the protein was quenched, reflecting population of the ligandprotein complex (Fig. $2 a$ ). The titration curve yields an equilibrium dissociation constant $\left(K_{\mathrm{d}}\right)$ of $25 \mu M$ for binding of the novel aminothiazole ligand to the $E$. coli KAS I protein. The affinity of this compound is similar to those of the known reference inhibitors thiolactomycin $(25 \mu M)$ and cerulenin $(3 \mu M)$ (Price et al., 2001). Further characterization of the complex was achieved by analytical ultracentrifugation experiments. Comparison of the sedimentation equilibrium of the ligand and of a mixture of ligand and protein shows a clear indication of complex formation (Fig. 2b). In addition, the solubility of the aminothiazole derivative was determined to be at least $100 \mu M$ in the buffer used for the binding assay.

\subsection{Crystallization of $E$. coli KAS I with bound aminothiazole ligand}

Crystallographic structure determination of E. coli KAS I with bound aminothiazole ligand was used to obtain molecular details of the interactions with its target. E. coli KAS I was prepared and crystallized as described previously (Olsen et al., 1999). A cocrystallization method was reported in the structure determination of E. coli KAS I with inhibitors (Price et al., 2001), but crystal soaking allows more efficient screening of a large number of compounds. Consequently, we established a soaking procedure for the crystals of $E$. coli KAS I with the reference inhibitor thiolactomycin. The resulting $F_{\mathrm{o}}-F_{\mathrm{c}}$ electron-density map from a data set at $1.5 \AA$ A (Table 1 ) clearly confirmed binding of thiolactomycin at the active site.

However, when crystal soaking was performed with the aminothiazole compound, the $F_{\mathrm{o}}-F_{\mathrm{c}}$ map from a $1.6 \AA$ data set showed only weak additional density around the active site that proved difficult to interpret, despite the similar affinities of thiolactomycin and the aminothiazole compound of around $25 \mu M$. We assume that the weak binding under crystalsoaking conditions was a consequence of the low solubility of the aminothiazole compound of $4.8 \mu M$ at the high ionic strength of the crystal soaking buffer $(20 \mathrm{~m} M$ Tris $\mathrm{pH} 7.5$, $2.3 \mathrm{M}$ ammonium sulfate, $1 \% \mathrm{DMSO}$ ) compared with at least $100 \mu M$ at low ionic strength as used in the assay buffer. Therefore, a new soaking system was evaluated with better solubility conditions. Finally, 30\% PEG 8000 proved to be a good low ionic strength surrogate for ammonium sulfate in the crystal-soaking buffer that had no adverse effect on diffraction quality after crystal soaking; a solubility of at least $100 \mu M$ was confirmed for this buffer condition (30\% PEG 8000, $0.1 \mathrm{M}$ ammonium sulfate, $0.1 M$ Tris- $\mathrm{HCl} \mathrm{pH}$ 7.5, $5 \mathrm{~m} M$ TCEP, $1 \%$ DMSO).

To exclude any effects of the change in solvent conditions on the protein, the apoprotein structures of crystals from ammonium sulfate-based and PEG 8000-based crystal soaking buffer were compared (see data sets in Table 1). These protein 
structures are virtually identical. Near the active site a rotation around $\chi_{2}$ is observed for the Phe392 side chain in three of the four chains in the asymmetric unit (monomers $B, C$ and $D$ ), which is indicative of the flexibility of this lid of the active site (see below). In monomer $C$, partial oxidation of the active-site Cys163 to sulfinic acid was noted in the PEG 8000-based crystal-soaking buffer. This is in contrast to the ammonium sulfate conditions, where no indication of oxidation was observed even after prolonged soaks. For subsequent soaks in PEG 8000, $5 \mathrm{~m} M$ TCEP was added to the soaking buffer as a reducing agent, which prevented oxidation of the active-site Cys163.

Crystal soaking with the aminothiazole compound in PEG 8000 in the presence of TCEP yielded a $1.35 \AA$ data set (Table 1) that revealed strong electron density for the bound ligand at the active site in the $F_{\mathrm{o}}-F_{\mathrm{c}}$ map (Fig. 3a). This data set provides the highest resolution structure of E. coli KAS I obtained to date, enabling unambiguous assignment of the ligand-binding mode, of protein side-chain conformations (such as Phe392) and the positioning of water molecules (such as $\mathrm{HOH} 2433$ ) around the active site to aid in interpretation of the structural information.

\subsection{Description of the interactions of the aminothiazole with the active-site residues}

In the crystal structure of the complex of $E$. coli KAS I with the aminothiazole compound, the ligand binds directly adjacent to the active-site residue Cys163 (Fig. 3a). The phenyl ring stretches into the narrow fatty-acid binding pocket, which is also the binding site for the inhibitor cerulenin. The thiazole moiety reaches out towards the more spacious malonyl-ACP binding pocket, which also harbours the thiolactomycinbinding site (Fig. 3b). This binding mode at the centre of the active site is distinct from those of the inhibitors thiolactomycin and cerulenin.

The strong and specific interaction of the aminothiazole with E. coli KAS I can primarily be attributed to three features (Fig. 4). (i) A close but noncovalent interaction is observed between the reactive active-site Cys163 and both the
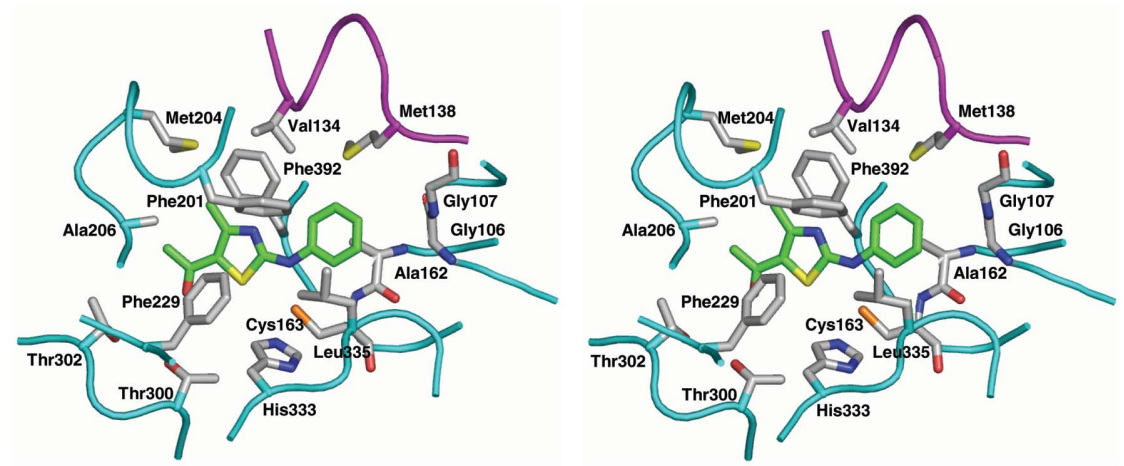

Figure 4

Stereo diagram of the interactions of the aminothiazole ligand at the active site of $E$. coli KAS I. The aminothiazole molecule is shown in green. Protein side chains or peptide bonds which interact with the ligand are shown in grey. The backbone trace is indicated in cyan for monomer $A$ and in magenta for monomer $B$. thiazole $\mathrm{S}$ atom and the exocyclic amine $\mathrm{N}$ atom, with distances of 3.3 and $3.1 \AA$, respectively. This indicates strong van der Waals interactions between the two $\mathrm{S}$ atoms and a short $\mathrm{NH} \cdots \mathrm{S}$ hydrogen bond between the Cys163 S atom and the exocyclic amine $\mathrm{N}$ atom. Such hydrogen bonds between an $\mathrm{N}$ and an $\mathrm{S}$ atom are not uncommon in proteins, but usually have a distance of 3.4-3.7 $\AA$ instead of $3.1 \AA$ (Gregoret et al., 1991). Similarly, the two $S$ atoms are also at a very close distance: a survey of noncovalent protein-ligand interactions indicates a preferred distance of two $\mathrm{S}$ atoms of $3.8 \AA$, with a distance of or below $3.3 \AA$ only seen in $1.5 \%$ of cases. These close interactions are possibly a consequence of the bidentate arrangement of the $\mathrm{S}$ and $\mathrm{N}$ atoms in the aminothiazole or of a negative charge at the active-site Cys163. (ii) Several aromatic amino acids show $\pi$-stacking interactions (Meyer et al., 2003) with the aromatic system of the aminothiazole. Phe392 is in a parallel displaced arrangement with the thiazole ring. This interaction stabilizes the conformation of the side chain of Phe392, which is only poorly defined in the apo form but becomes well resolved in the complex structure. T-shaped stacking interactions are found between Phe229 and the thiazole ring, as well as between Phe201 and the phenyl ring of the ligand. An additional $\pi$-stacking interaction is observed between the peptide bond of Ala162 and Cys163 and the phenyl ring of the aminothiazole. (iii) The aminothiazole molecule has an excellent shape complementarity to the E. coli KAS I active site and is almost completely buried. This is particularly true for the phenyl moiety, which is in close interaction with a number of hydrophobic residues: Phe201, Leu335, Ala162, Met138, Val134 and the peptide backbone at Gly106, Gly107 and Ala162. Met138 and Val134 are contributed by chain $B$ of the dimer. A slight torsion of $20^{\circ}$ away from coplanarity is observed between the thiazole and the phenyl ring (Fig. $3 b$ ), presumably to optimize the steric fit of the molecule to the active site. Additional hydrophobic interactions are provided by the methyl substituent of the thiazole ring with residues Phe201, Met204, Ala206 and Phe392.

The acetyl moiety of the aminothiazole ligand is significantly less well defined than the remainder and no obvious density is observed in the $F_{\mathrm{o}}-F_{\mathrm{c}}$ map for the carbonyl $\mathrm{O}$ atom and methyl $\mathrm{C}$ atom of the acetyl group (Fig. 3a). The chemical structure and the purity of the aminothiazole were checked and verified using LC-MS and NMR spectroscopy. Consequently, the lack of electron density must indicate rotational flexibility of the acetyl group, rather than a coplanar conformation with the rest of the molecule. Indeed, rather few interactions are found between the protein and the acetyl group of the ligand. Only the Ala206 side chain is in proximity to the methyl $\mathrm{C}$ atom, with a $3.8 \AA$ distance; all other protein atoms are $4.5 \AA$ or further away. The carbonyl $\mathrm{O}$ atom points towards Thr300 and Thr302, but the distances are too long for strong hydrogenbonding interactions $(3.9$ and $3.6 \AA$, 
respectively). Instead, a well defined water molecule ( $\mathrm{HOH} 2433)$ is hydrogen bonded to the side-chain $\mathrm{O}$ atoms of Thr300 (2.9 $\AA$ distance) and further water molecules in the active site (Fig. $3 a$ ). If the acetyl group is positioned coplanar with the remainder of the aminothiazole, the carbonyl $\mathrm{O}$ atom would need to displace this water molecule, while being too far

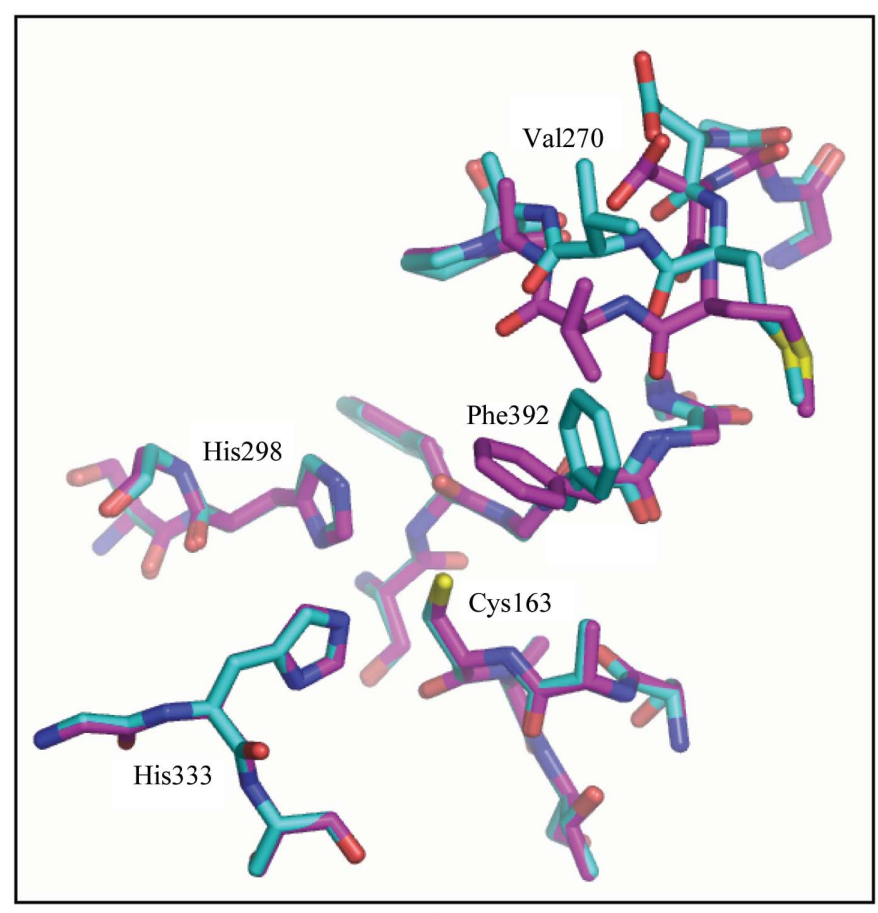

(a)

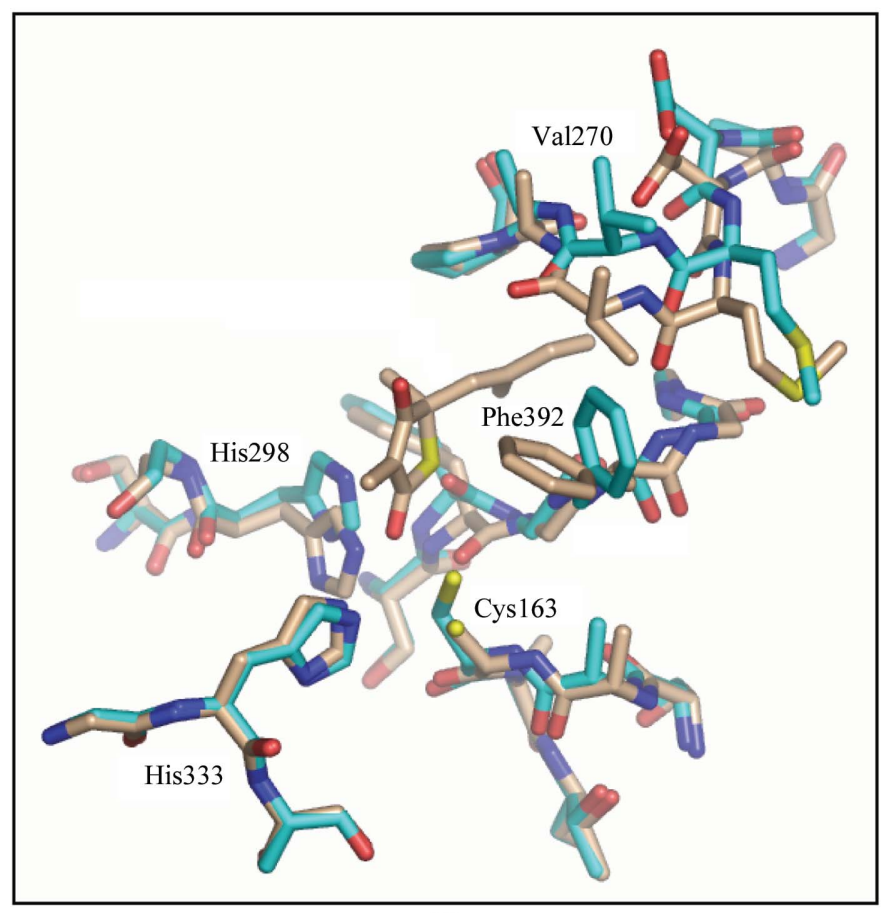

(b) away from Thr300 to provide a good alternative hydrogenbond interaction. If the acetyl group is instead in an out-ofplane orientation, it avoids the energetic penalty of removing this water molecule at the expense of reduced participation in the delocalized electron system. The electron density around the acetyl moiety of the ligand is thus interpreted as the mutally exclusive presence of the water molecule $\mathrm{HOH} 2433$ (with an occupancy of 0.5 ) or a coplanar acetyl moiety of the ligand (with an occupancy of 0.5 ).

\subsection{Ligand-induced fit of the active-site conformation}

In the E. coli KAS I apo structure, there are four molecules in the asymmetric unit of the crystal with very similar overall structure. However, the side chain of Phe392 and a neighbouring loop (Asp268-Pro272, particularly Val270) near the active site exhibit high temperature factors in all molecules and differ in conformation (Fig. $5 a$ ). In monomer $A$, the side chain of Phe392 is rotated around $\chi_{1}$ such that the access to the active site is open. This conformation can only be achieved by an evasive movement of the neighbouring loop around Val270. In contrast, in monomers $B, C$ and $D$ the phenyl ring closes the active-site pocket together with the loop around Val270.

Binding of thiolactomycin to monomer $A$ results in an induced fit: Phe392 and the loop around Val270 in monomer $A$ are now in the same closed conformation (Fig. 5b) as seen in the apo structures of the other monomers.

The binding of the aminothiazole ligand to monomer $A$, in contrast, does not change the active-site geometry: Phe392 and the loop around Val270 remain in the open conformation

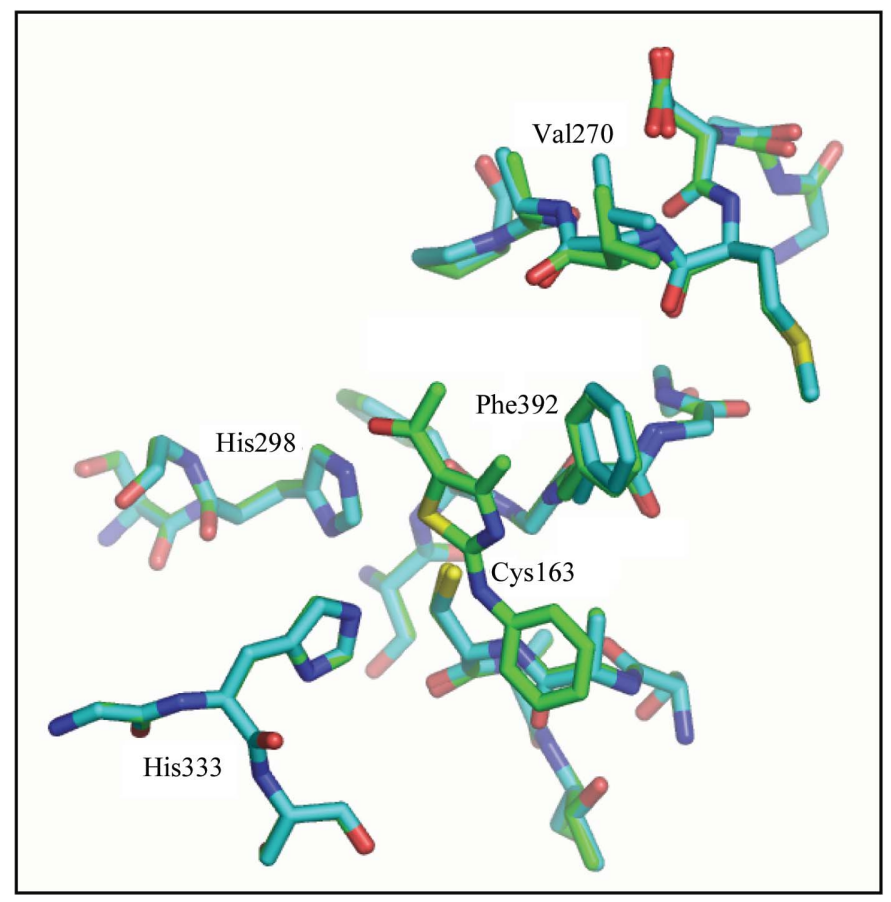

(c)

Figure 5

Conformational rearrangements in the active-site regions of $E$. coli KAS I. Superposition of monomer $A$ (cyan) of the apo enzyme with (a) apo enzyme, monomer $B$ (magenta), showing the difference in the active-site geometry of the different monomers in the apo structure, $(b)$ enzyme with bound thiolactomycin, monomer $A$ (gold), showing the induced fit upon binding of thiolactomycin, and $(c)$ enzyme with bound aminothiazole ligand, monomer $A$ (green), showing the absence of significant conformational changes upon binding of the aminothiazole ligand. 
(Fig. 5c) as in the apo structure of monomer $A$. This underlines the perfect steric fit of the aminothiazole ligand to the activesite pocket. The advantageous $\pi$-stacking interactions of Phe392 and the thiazole ring result in an improved temperature factor of the side chain of Phe392 compared with the apo structure. This interaction with an open active site is similar to the binding of the natural inhibitor platensimycin to E. coli KAS II, which requires the same arrangement of the equivalent residue Phe400 in E. coli KAS II (Wang et al., 2006).

However, incubation with the aminothiazole ligand does not result in an induced fit for the other three monomers: Phe392 and the loop around Val270 remain unchanged in the closed conformation. The open active-site conformation would be required to provide space for the thiazole ring and its methyl substituent of the ligand and accordingly no ligand can be detected at the active site in these three monomers. From the crystal structure of the monomers and their interactions, there is no obvious explanation why Phe392 and the loop around Val270 are in the closed conformation in monomers $B, C$ and $D$ and why the open conformation could not be induced. We have to assume that the causes of the different conformations of the monomers are hidden in the subtleties of the overall protein structure.

\subsection{Analysis of the binding mode of aminothiazole and implications for drug discovery}

The binding mode of the aminothiazole ligand is particularly surprising with respect to its resemblance to thiolactomycin (Fig. 1). Both ligands can be superimposed with high shape similarity, bringing the $\mathrm{S}$ atoms into equivalent positions (Fig. 6). From such ligand-based shape matching one would have considered the aminothiazole compound to be a thiolactomycin analogue. In silico docking of the aminothiazole compound into the apo structure of E. coli KAS I indeed suggests an identical binding mode to that of thiolactomycin for those protein monomers with the closed conformation of Phe392 $(B, C$ and $D)$. In protein monomer $A$ with the open conformation of Phe392, however, the experimentally determined novel binding mode is correctly predicted. This exemplifies the high predictive value that can be achieved by computational methods for the understanding of proteinligand interactions, but also highlights the critical importance of conformationally representative protein structure. Computational methods reach their limits where ligand binding is accompanied by significant yet unknown changes in the protein structure.

To evaluate whether a hit from a screen is a good starting point for further optimization, the potency of the new ligand should be weighted by its molecular mass. This is achieved by calculation of the ligand efficiency: the free energy of binding per non-H atom of the ligand (Hopkins et al., 2004). With a binding affinity of $25 \mu \mathrm{M}$ and a molecular mass of 232, the aminothiazole ligand has a ligand efficiency of $1.63 \mathrm{~kJ} \mathrm{~mol}^{-1}$ per non-H atom. This value is well within the range of established drug molecules for drug targets. Furthermore, the aminothiazole scaffold is chemically tractable and provides options for synthetic chemistry modifications. The structural information can immediately be used to support the rational design of improved inhibitors. Owing to its central binding at the active site, the ligand can expand into both the fatty-acid and the malonyl-ACP binding pockets (Fig. $3 b$ ). The aminothiazole ligand therefore presents an excellent starting point for lead-generation chemistry.

The phenyl moiety of the aminothiazole ligand occupies part of the fatty acid-binding pocket with an excellent fit. This binding pocket offers the possibility of extending the ligand with an aliphatic moiety similar to that observed in cerulenin (Fig. 3b). However, proper positioning of the exit vector would require significant shifts of the phenyl moiety of the ligand. This could be possible since the molecular basis for the interaction is here primarily hydrophobic, with only a limited contribution from directed interactions such as hydrogen bonds. Consequently, a great variety of hydrophobic side chains could fit to this geometry and further exploration of the fatty acid binding pocket could be an option to improve the binding affinity.

The methyl substituent on the thiazole ring offers a second exit vector, but the space for extension is restricted by Met204 which is in proximity. The most promising exit vector is the acetyl moiety of the ligand. It points directly into the large water-filled malonyl-ACP binding pocket. Furthermore, its structural environment indicates less than optimal interactions with the protein (see above). One could therefore conceive substituents that could properly substitute for water $\mathrm{HOH} 2433$ as a hydrogen-bonding partner to Thr300 or explore further hydrogen-bonding opportunities within the polar malonyl-ACP binding pocket. A further obvious possi-

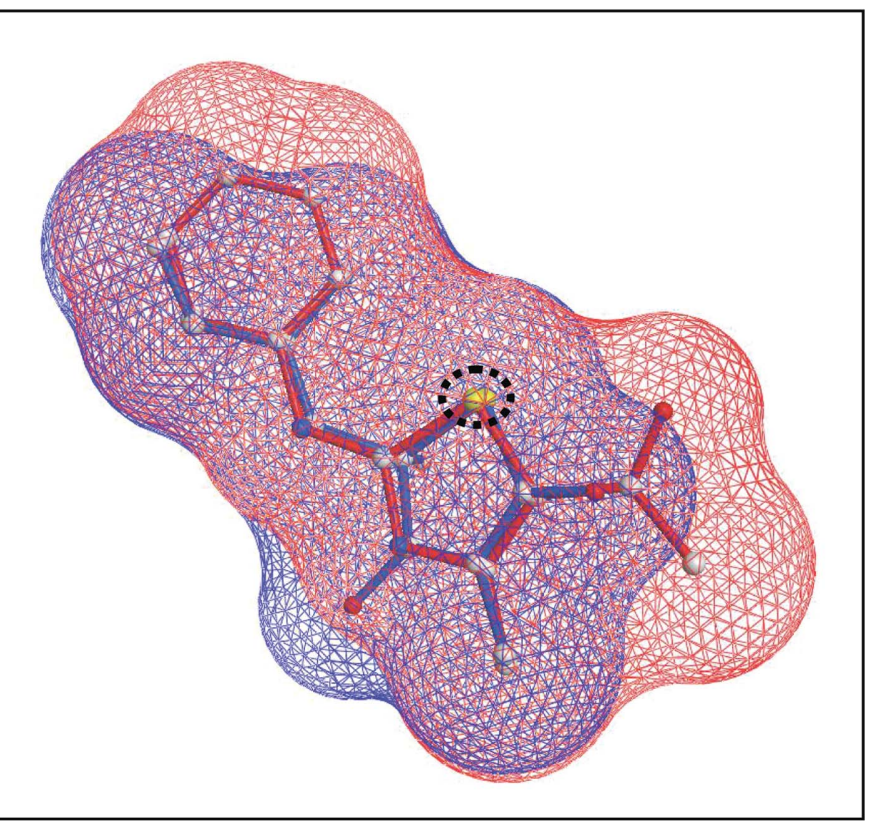

\section{Figure 6}

Similarity of the aminothiazole compound to thiolactomycin. Shapematching superposition of the aminothiazole compound (red) with thiolactomycin (blue) results in a high shape Tanimoto score of 0.73 and a colour score of 0.32 . The dashed circle indicates the exact superposition of the $\mathrm{S}$ atoms of the two compounds. 
bility is a link from the aminothiazole to the isoprenoid moiety of thiolactomycin in order to exploit its binding to the hydrophobic side pocket of the malonyl-ACP binding site. Furthermore, improvement of the physicochemical properties of the molecule could be achieved by modifications at the acetyl substituent. We therefore regard the aminothiazole molecule as an excellent starting point for optimization to a highly active and novel broadband antibacterial drug.

We thank Josef Schneider and Louis Allemann for analytical investigation of the aminothiazole sample by NMR and LC-MS, respectively. Helpful discussions with Daniel Schlatter, Ralf Thoma, Jörg Benz and Armin Ruf are gratefully acknowledged. Thanks to the staff at the SLS synchrotron (Villigen, Switzerland) for professional support.

\section{References}

Barrett, C. T. \& Barrett, J. F. (2003). Curr. Opin. Biotechnol. 14, 621-626.

Bhargava, H. N. \& Leonard, P. A. (1996). Am. J. Infect. Control, 24, 209-218.

Birdsall, B., King, R., Wheeler, M., Lewis, C. J., Goode, S., Dunlap, R. \& Roberts, G. (1983). Anal. Biochem. 132, 353-361.

Collaborative Computational Project, Number 4 (1994). Acta Cryst. D50, 760-763.

D’Agnolo, G., Rosenfeld, I. S., Awaya, J., Omura, S. \& Vagelos, P. R. (1973). Biochim. Biophys. Acta, 326, 155-166.

Daines, R. A., Pendrak, I., Sham, K., Van Aller, G. S., Konstantinidis, A. K., Lonsdale, J. T., Janson, C. A., Qiu, X., Brandt, M., Khandekar, S. S., Silverman, C. \& Head, M. S. (2003). J. Med. Chem. 46, 5-8.

DeLano, W. L. (2002). The PyMOL User's Manual. DeLano Scientific, San Carlos, CA, USA.

Douglas, J. D., Senior, S. J., Morehouse, C., Phetsukiri, B., Campbell, I. B., Besra, G. S. \& Minnikin, D. E. (2002). Microbiology, 148, 3101-3109.

Edwards, P., Nelsen, J. S., Metz, J. G. \& Dehesh, K. (1997). FEBS Lett. 402, 62-66.

Eftink, M. (1997). Methods Enzymol. 278, 221-257.

Garwin, J. L., Klages, A. L. \& Cronan, J. E. (1980). J. Biol. Chem. 255 , 3263-3265.

Gerber, P. R. (1992). Biopolymers, 32, 1003-1017.

Gregoret, L. M., Rader, S. D., Fletterick, R. J. \& Cohen, F. E. (1991). Proteins, 9, 99-107.

Hayashi, T., Yamamoto, O., Sasaki, H., Kawaguchi, A. \& Okazaki, H. (1983). Biochem. Biophys. Res. Commun. 115, 1108-1113.

He, X., Reeve, A. M., Desai, U. R., Kellogg, G. E. \& Reynolds, K. A. (2004). Antimicrob. Agents Chemother. 48, 3093-3102.

He, X. \& Reynolds, K. A. (2002). Antimicrob. Agents Chemother. 46, 1310-1318.

Heath, R. J. \& Rock, C. O. (2002). Nat. Prod. Rep. 19, 581-596.

Heath, R. J., White, S. W. \& Rock, C. O. (2001). Prog. Lipid Res. 40, 467-497.
Hopkins, A. L., Groom, C. R. \& Alex, A. (2004). Drug Discov. Today, 9, 430-431.

Huang, W., Jia, J., Edwards, P., Dehesh, K., Schneider, G. \& Lindqvist, Y. (1998). EMBO J. 17, 1183-1191.

Kabsch, W. (1993). J. Appl. Cryst. 26, 795-800.

Khandekar, S. S., Daines, R. A. \& Lonsdale, J. T. (2003). Curr. Protein Pept. Sci. 4, 21-29.

Lai, C.-Y. \& Cronan, J. E. (2003). J. Biol. Chem. 278, 51494-51503.

McFadden, J. M., Medghalchi, S. M., Thupari, J. N., Pinn, M. L., Vadlamudi, A., Miller, K. I., Kuhajda, F. P. \& Townsend, C. A. (2005). J. Med. Chem. 48, 946-961.

Meyer, E. A., Castellano, R. K. \& Dierderich, F. (2003). Angew. Chemie Int. Ed. 42, 1210-1250.

Miyakawa, S., Suzuki, K., Noto, T., Harada, Y. \& Okazaki, H. (1982). J. Antibiot. (Tokyo), 35, 411-419.

Moche, M., Schneider, G., Edwards, P., Dehesh, K. \& Lindqvist, Y. (1999). J. Biol. Chem. 274, 6031-6034.

Morita, Y. S., Paul, K. S. \& Englund, P. T. (2000). Science, 288, 140-143.

Murshudov, G. N., Vagin, A. A., Lebedev, A., Wilson, K. S. \& Dodson, E. J. (1999). Acta Cryst. D55, 247-255.

Nie, Z., Perretta, C., Lu, J., Su, Y., Margosiak, S., Gajiwala, K. S., Cortez, J., Nikulin, V., Yager, K. M., Appelt, K. \& Chu, S. (2005). J. Med. Chem. 48, 1596-1609.

Nishida, I., Kawaguchi, A. \& Yamada, M. (1986). J. Biochem. (Tokyo), 99, 1447-1454.

Noto, T., Miyakawa, S., Oishi, H., Endo, H. \& Okazaki, H. (1982). J. Antibiot. (Tokyo), 35, 401-410.

Olsen, J. G., Kadziola, A., Siggaard-Andersen, M., Chuck, J.-A., Larsen, S. \& von Wettstein-Knowles, P. (1995). Protein Pept. Lett. 1, 246-251.

Olsen, J. G., Kadziola, A., von Wettstein-Knowles, P., SiggaardAndersen, M. \& Larsen, S. (2001). Structure, 9, 233-243.

Olsen, J. G., Kadziola, A., von Wettstein-Knowles, P., SiggaardAndersen, M., Lindqvist, Y. \& Larsen, S. (1999). FEBS Lett. 460, 46-52.

Payne, D. J. (2004). Drug News Perspect. 17, 187-194.

Perrakis, A., Morris, R. \& Lamzin, V. S. (1999). Nature Struct. Biol. 6, 458-463.

Price, A. C., Choi, K.-H., Heath, R. J., Li, Z., White, S. W. \& Rock, C. O. (2001). J. Biol. Chem. 276, 6551-6559.

Rock, C. O. \& Jackowski, S. (2002). Biochem. Biophys. Res. Commun. 292, 1155-1166.

Rosenfeld, I. S., D'Agnolo, G. \& Vagelos, P. R. (1973). J. Biol. Chem. 248, 2452-2460.

Sakya, S. M., Suarez-Contreras, M., Dirlam, J. P., O’Connell, T. N., Hayashi, S. F., Santoro, S. L., Kamicker, B. J., George, D. M. \& Ziegler, C. B. (2001). Bioorg. Med. Chem. Lett. 11, 2751-2754.

Schuck, P. (1994). Prog. Colloid Polym. Sci. 94, 1-13.

Smith, S., Witkowski, A. \& Joshi, A. K. (2003). Prog. Lipid Res. 42, 289-317.

Vance, D., Goldberg, I., Mitsuhashi, O. \& Bloch, K. (1972). Biochem. Biophys. Res. Commun. 48, 649-656.

Waller, R. F., Keeling, P. J., Donald, R. G., Striepen, B., Handman, E., Lang-Unnasch, N., Cowman, A. F., Besra, G. S., Roos, D. S. \& McFadden, G. I. (1998). Proc. Natl Acad. Sci. USA, 95, 1235212357.

Wang, J. et al. (2006). Nature (London), 441, 358-360. 